\title{
Spatial Distance Effect of Bienzymes on the Efficiency of Sequential Reactions in a Microfluidic Reactor Packed with Enzyme-immobilized Microbeads
}

\author{
Jinseok HEO \\ Department of Chemistry, The State University of New York College at Buffalo, 1300 Elmwood Ave., Buffalo, \\ NY 14222, USA
}

\begin{abstract}
Three different configurations of microfluidic reactors packed with enzyme-bearing microbeads were examined to show that the overall efficiency of coupled enzyme-catalyzed reactions depends on the spatial relationship of two enzymes immobilized on the bead surfaces. The spatial distances of glucose oxidase (GOx) and horseradish peroxidase (HRP) enzymes were controlled by using microbeads as a supporting matrix for immobilizing the two enzymes and packing them in two microfluidic chambers. A microreactor packed with microbeads coimmobilized with the two enzymes showed a better overall reaction efficiency than the other two reactors, where the two enzymes were spatially distant, under a flow condition. These results are ascribed to the reduced diffusional loss of an intermediate product in the bienzyme-coimmobilized microreactor. Furthermore, the inhibition of the GOx enzyme by $\mathrm{H}_{2} \mathrm{O}_{2}$, an intermediate product, can be eliminated by quickly converting $\mathrm{H}_{2} \mathrm{O}_{2}$ to a final non-inhibiting product in the bienzyme-coimmobilized microreactor.
\end{abstract}

Keywords Microfluidic reactor, sequential enzyme reaction, enzyme immobilization

(Received July 21, 2014; Accepted August 13, 2014; Published October 10, 2014)

\section{Introduction}

Here, we present three different configurations of microfluidic reactors that carry out sequential, two-step, enzyme-catalyzed reactions under flow conditions (Scheme 1). In all of these configurations, substrate molecules are delivered to the reactor zone by a pressure-driven flow, encountered by the beadimmobilized enzymes, and converted into products. We find that the overall rate of product generation depends on the spatial relationship of the two enzymes. In reactor I, both enzymes were co-immobilized on a single set of beads. In reactor II, the enzyme required to catalyze the first step of the reaction sequence was immobilized on one set of beads, and the enzyme necessary to catalyze the second step was immobilized on a second set of beads. The two-step reaction was then carried out by sequentially passing the substrate over the two sets of beads. In reactor III, the two sets of enzyme-immobilized beads were mixed. Experiments showed that the reactor I configuration resulted in a more effective substrate turnover. Our results clearly demonstrate the usefulness of a microfabricated system and enzyme-immobilized beads in designing an efficient, multi-step microfluidic reactor based on immobilized enzymes.

Immobilized-enzymes have been widely used in various areas, including the food industry, pharmaceutical medicine, biomedical engineering, biofuel production, and environmental remediation because of the convenient recovery process of

To whom correspondence should be addressed.

E-mail: heoj@buffalostate.edu enzymes from solution and the potential reuse of the enzymes. ${ }^{1}$ Recently, microfabrication technology has opened up a new era in the applications of immobilized enzymes.,3 Microfluidic reactors based on immobilized enzymes have been broadly applied for proteomics, ${ }^{4}$ drug discovery, ${ }^{5-7}$ small scale synthesis of compounds, ${ }^{8-12}$ kinetic study of immobilized enzymes, ${ }^{13-15}$ and biosensing. ${ }^{16-18}$ Microfluidic reactors have been an attractive

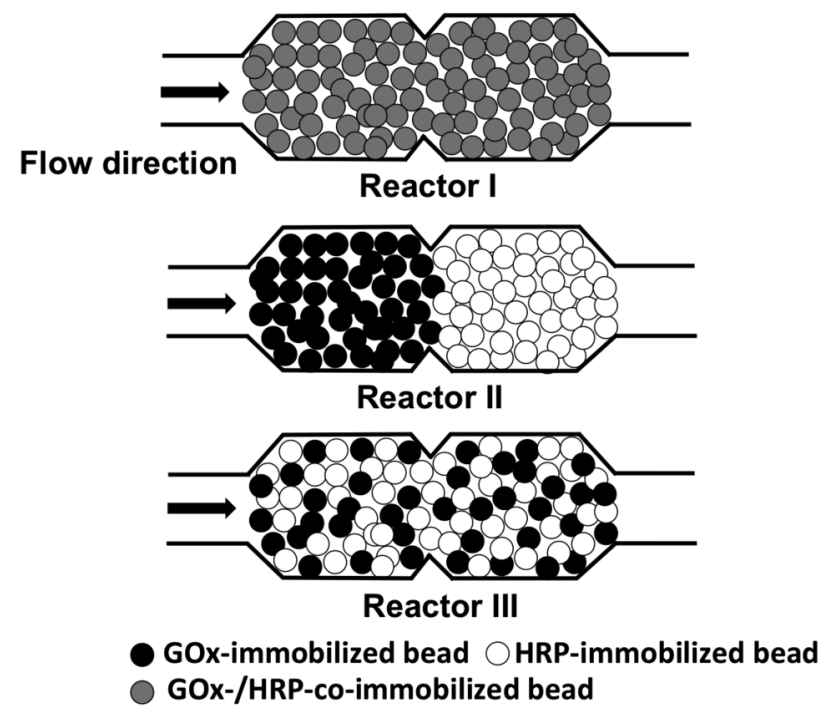

Scheme 1 Three different configurations of microfluidic reactors. 
platform for these applications, because they consume small amounts of reagents, and their dimensions are small enough to handle or mimic a biological system at a cellular level.

While many of the previous applications rely on a single-step enzyme reaction, the applications of multiple immobilized enzymes in the microfluidic reactor to perform multi-step, consecutive reactions have been growing. ${ }^{11,18-22}$ This is partly because there are increasing interests in using multiple enzymes for synthesizing new compounds and in developing synthetic biology. It has been previously reported that multiple enzymes can be either co-immobilized on a surface ${ }^{19,20}$ or within a matrix $^{18}$ in proximity, or be immobilized separately ${ }^{11,19,21,22}$ on discrete zones of a microreactor, which are assigned for each of the enzymes. Intuitively, it is expected that the spatial distances between the multiple enzymes will affect the overall efficiency of the coupled enzyme reactions. In the early 1970's Mosbach noted the importance of the spatial relationship of bienzymes on the overall reaction efficiency. ${ }^{23,24}$ However, their study was performed using a large scale of enzyme-immobilized polymeric matrices under a non-fluidic condition. Therefore, it is difficult to adopt their results in a small-scale reaction system under a fluidic condition, at which we are aiming.

Our report demonstrates the relative effectiveness of different catalyst configurations for carrying out sequential reactions within microfluidic systems. Here, polymeric microbeads were selected as a supporting matrix for immobilizing two different enzymes. These microbeads can provide a large surface-tovolume ratio for immobilizing enzymes, efficiently mix laminar flows, and reduce the diffusional distances of substrate molecules to the enzymes on the bead surfaces. More importantly, they can be conveniently used to control the spatial distances of multiple enzymes, as demonstrated in this report. The sequential enzymatic reactions shown in Scheme 2 were used to examine the effect of spatial distances of the two enzymes on the substrate turnover. The experimental results showed that the overall reaction efficiency of the reactor $\mathbf{I}$ in Scheme 1 was much higher than the other two reactors (II and III in Scheme 1). This report will discuss the results in terms of two effects: the molecular mass transport of an intermediate product and the inhibition of glucose oxidase (GOx) enzyme by the intermediate product generated during the sequential enzyme reactions.

\section{Experimental}

\section{Reagents and chemicals}

Poly(dimethylsiloxane) (PDMS) prepolymer (Sylgard 184) was purchased from Dow Corning Co. (Midland, MI). Positive photoresist (AZ P4620) and developer solution (AZ 421K) were purchased from AZ Electronic Materials. (Branchburg, NJ). Biotin amidocaproyl-labeled glucose oxidase (biotin-GOx, $M_{\mathrm{W}}=160 \mathrm{kDa}$, from Aspergillus niger), biotin amidocaproyllabeled peroxidase (biotin-HRP, type VI, $M_{\mathrm{W}}=44 \mathrm{kDa}$, from Horseradish), horseradish peroxidase (HRP, type VI-A, from Horseradish), glucose, biotin-fluorescein, $\mathrm{H}_{2} \mathrm{O}_{2}$ (analytical reagent, $30 \mathrm{wt} \%$ ), and resorufin were purchased from Sigma Chemical Co. (St. Louis, MO). Avidin-coated microbeads (SuperAvidin ${ }^{\mathrm{TM}}$ Coated Microspheres, $9.95 \mu \mathrm{m}$ diameter) were obtained from Bangs Laboratories, Inc. (Fishers, IN). Phosphate buffered saline (PBS) solution was prepared by dissolving $130 \mathrm{mmol}$ of $\mathrm{NaCl}, 2.7 \mathrm{mmol}$ of $\mathrm{KCl}, 10 \mathrm{mmol}$ of $\mathrm{Na}_{2} \mathrm{HPO}_{4}$ (Sigma) and $1.8 \mathrm{mmol}$ of $\mathrm{KH}_{2} \mathrm{PO}_{4}($ Sigma) in $1 \mathrm{~L}$ of deionized water and adjusting the $\mathrm{pH}$ to 7.4 with $\mathrm{HCl}$ or $\mathrm{NaOH}$. Similarly, $50 \mathrm{mM}$ Tris buffer solution was prepared by dissolving

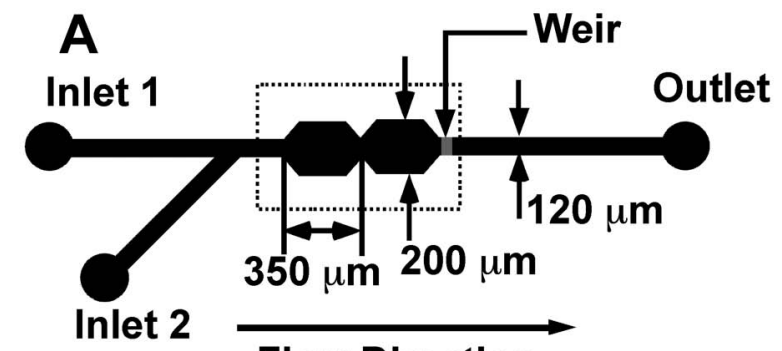

Flow Direction

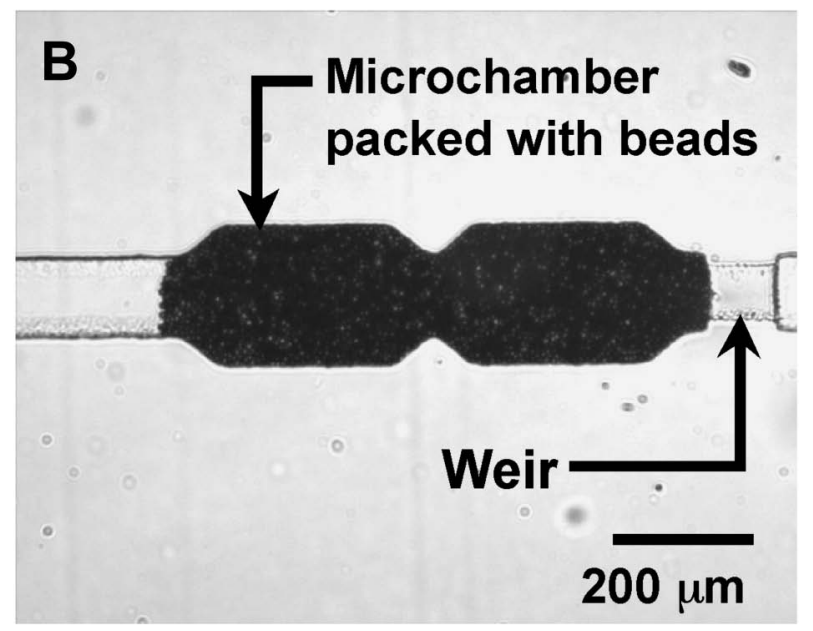

Fig. 1 (A) Schematic diagram of a microfluidic reactor used for tests. (B) Optical micrograph showing the region of the device enclosed within the black dotted box in Fig. 1(A).

$41.4 \mathrm{mmol}$ of tris(hydroxymethyl)aminomethane hydrochloride (Sigma) and $8.6 \mathrm{mmol}$ of the base form in $1 \mathrm{~L}$ of deionized water and adjusting the $\mathrm{pH}$ to 7.4 with $\mathrm{HCl}$ or $\mathrm{NaOH}$. Amplex Red ( $N$-acetyl-3,7-dihydroxyphenoxazin-3-one) was purchased from Invitrogen, Inc. (Eugene, OR). All aqueous solutions were prepared using $18 \mathrm{M} \Omega-\mathrm{cm}$ water.

\section{Preparation of microfluidic reactors packed with enzyme-} immobilized beads

The microfluidic bioreactors used in this study were fabricated using a previously reported procedure (see the procedures in Supporting Information). ${ }^{22}$ A schematic illustration of the device design is shown in Fig. 1A. Three different sets of enzyme-bearing beads (HRP-bearing beads, GOx-bearing beads, and GOx-/HRP-bearing beads) were prepared using biotinylated enzymes and avidin-coated microbeads (see the procedures in Supporting Information). The beads co-immobilized with the GOx and HRP enzymes had a roughly the same mole numbers of GOx and HRP enzymes on their surfaces.

Enzyme-modified microbeads $\left(3.66 \times 10^{5}\right.$ beads $/ \mathrm{mL}$ in buffer $)$ were introduced to the microreactor via inlet 2 (Fig. 1A) using a syringe. PBS buffer was simultaneously injected into inlet 1 to control the rate of bead deposition in the microchambers. Reactor I (Scheme 1) was prepared by introducing microbeads co-conjugated with both GOx and HRP into both microchambers. Reactor II was prepared by first introducing HRP-conjugated microbeads into the chamber on the right side of the device and then GOx-conjugated microbeads were introduced into the chamber on the left. Reactor III was prepared by introducing a bead solution, which was prepared by mixing GOx-conjugated 

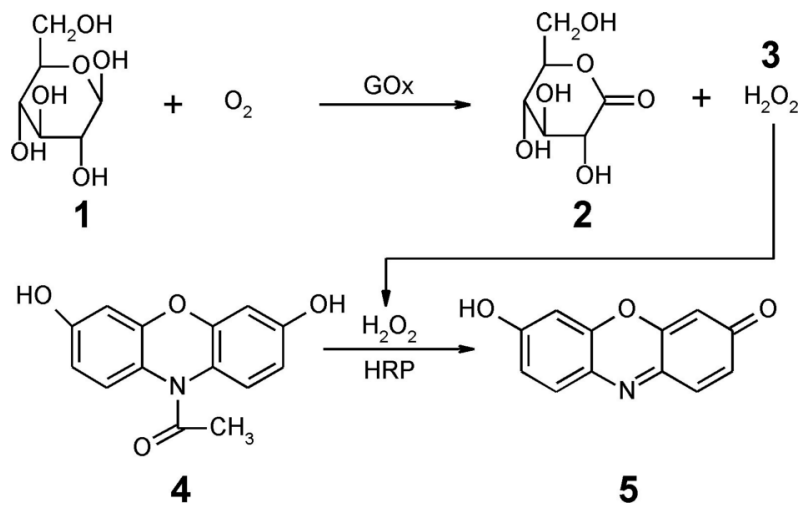

Scheme 2 Two-step, sequential enzyme reactions employed in microfluidic reactors.

and HRP-conjugated microbeads in a 1:1 volume ratio, into all microchambers.

\section{Test of microfluidic reactors}

A $0.500 \mathrm{M}$ glucose stock solution was prepared in Tris buffer ( $\mathrm{pH}$ 7.4) and stored at room temperature for one day prior to use. The stock solution was diluted with Tris buffer to prepare lower concentrations. Amplex Red solutions $(40 \mathrm{mM})$ were prepared in anhydrous DMSO and stored in $20 \mu \mathrm{L}$ aliquots at $-20^{\circ} \mathrm{C}$ until just before use. A solution containing $1.00 \mathrm{mM}$ glucose and $0.80 \mathrm{mM}$ Amplex Red in Tris buffer ( $\mathrm{pH}$ 7.4) was pumped through inlet 1 at a flow rate of $0.5 \mu \mathrm{L} / \mathrm{min}$ using a syringe pump (PHD 2000, Harvard Apparatus, Hollistion, MA) until the solution emerged from inlet 2 (Fig. 1A). Next, the opening of inlet 2 was plugged with a Teflon stopper, and then the glucose test solution was introduced into inlet 1 at a flow rate of $0.20,0,15,0.10$ and, $0.05 \mu \mathrm{L} / \mathrm{min}$, respectively. The fluorescent product (resorufin) of the coupled reactions (5 in Scheme 2) was observed $0.17 \mathrm{~mm}$ downstream of the end of the microreactor using an epifluorescence microscope (Nikon Eclipse TE 300, Nikon Co., Tokyo, Japan) equipped with a mercury lamp and a cube-type band-pass filter (XF-102-2 filter set, Omega Optical, Inc., Brattleboro, VT). Using a 16-bit gray-scale CCD camera (Photometrics Ltd., Tucson, AZ), fluorescence micrographs were acquired every $30 \mathrm{~s}$ for up to 20 min until a steady state fluorescence signal was reached. The integration time for all fluorescence micrographs was $0.7 \mathrm{~s}$, and the microdevices were only illuminated during data acquisition to minimize photobleaching. The test outlined above was repeated two more times using independently prepared microfluidic reactors.

\section{Results and Discussion}

Preparation of microfluidic reactors packed with enzymeimmobilized beads

Figure 1A is a schematic illustration of the microfluidic device used in these experiments, and Fig. 1B is an optical micrograph showing the region of the device enclosed within the dotted lines in Fig. 1A. The microchambers and channels were $\sim 22 \mu \mathrm{m}$ high, which was measured from the photoresist master using a profilometer; the remaining dimensions are provided in Fig. 1A. The volume of each of the two microchambers was $\sim 1.5 \mathrm{~nL}$. The gap between the top of the weir structure (Fig. 1B) and the floor of the channel was $6 \mu \mathrm{m}$, so it was able to retain the $10 \mu \mathrm{m}$-diameter beads used to immobilize the enzymes. The microbeads were packed into the microchambers using a syringe. The void fraction of a bead-packed microchamber of the sort used here has been estimated to be $0.5 .^{15,25}$ This is reasonable, considering that the packing fraction of perfect hexagonal close packing is 0.74 . Taking the void fraction into account, the estimated number of microbeads packed in each microchamber is 1350 .

The maximum binding capacities of avidin-coated beads for biotin-GOx and biotin-HRP enzymes were measured independently (Fig. S1, Supporting Information). Only $\sim 50 \%$ of the biotin-binding sites on the avidin-coated beads were available for each of the enzymes. These results suggest that by considering the protein foot areas of GOx $\left(50 \mathrm{~nm}^{2}\right)^{26}$ and HRP $\left(20 \mathrm{~nm}^{2}\right)^{27}$ to be comparable to that of avidin $\left(34 \mathrm{~nm}^{2}\right),{ }^{21}$ only one out of two biotin-binding sites of an avidin molecule (note that we assumed only two biotin-binding sites are present for one avidin molecule sitting on a bead surface, because two other binding sites are oriented toward the bead surface) is available for a biotin-GOx or biotin-HRP enzyme molecule. Thus, only one molecule of biotin-GOx or biotin-HRP can be bound to one avidin molecule.

After this confirmation, the GOx-bearing beads and the HRPbearing beads, which were fully covered by the GOx and HRP enzymes, respectively, were prepared by incubating the avidin beads with sufficient amounts of biotin-GOx and biotin-HRP, respectively. The surface concentrations of GOx and HRP enzymes prepared on each set of microbeads are approximately the same if the avidin sites of bead surfaces are saturated with the enzymes, because the avidin molecule on a bead surface cannot accommodate more than one molecule of biotin-GOx or biotin-HRP. The beads co-immobilized with biotin-GOx and biotin-HRP were prepared by incubating the avidin beads with a solution consisting of biotin-GOx and biotin-HRP enzymes in one-to-one mole ratio. The total mole numbers of biotinylated enzymes added were sufficient to fully cover the avidin molecules on the beads. In this manner the avidin molecules could be covered with equal mole numbers of biotin-GOx and biotin-HRP enzymes. It is expected that the affinities of biotin-GOx and biotin-HRP are similar to each other, because a spacer group $(\mathrm{C} 11)$ is present between the biotin and the enzymes, and so the affinity of biotinylated enzymes to avidin is less likely to be affected by the enzymes.

A potential problem of steric hindrance between GOx and HRP enzymes on a bead surface was examined. Only $\sim 62 \%$ of the surface area of each bead is occupied with avidin, which is estimated from the surface area and concentration of avidin $\left(34 \mathrm{~nm}^{2}\right.$ and $5.72 \times 10^{6}$ molecules/bead). If half of the avidin molecules are occupied by biotin-GOx and the other half by biotin-HRP, then the two enzymes cover $\sim 64 \%$ of the bead surface. This means that even at maximum coverage there is substantial free area remaining on the bead surface, and therefore the enzymes are not likely to sterically interfere with one another. This conclusion was supported by our observation that the order of immobilization for the beads supporting both enzymes (first GOx and then HRP, first HRP and then GOx, or simultaneous co-immobilization of both) did not affect their activity (Fig. S2, Supporting Information).

It has previously been reported that PDMS channels having aspect ratios (width/height) of $\sim 80$ are subject to bulging, and that this effect can bias fluorescence measurements obtained at different flow rates. ${ }^{28}$ However, no change in fluorescence was observed at a flow rate of up to $1.00 \mu \mathrm{L} / \mathrm{min}$ in a microfluidic reactor packed with microbeads (Fig. S3, Supporting Information). This is because we fabricated a microfluidic 

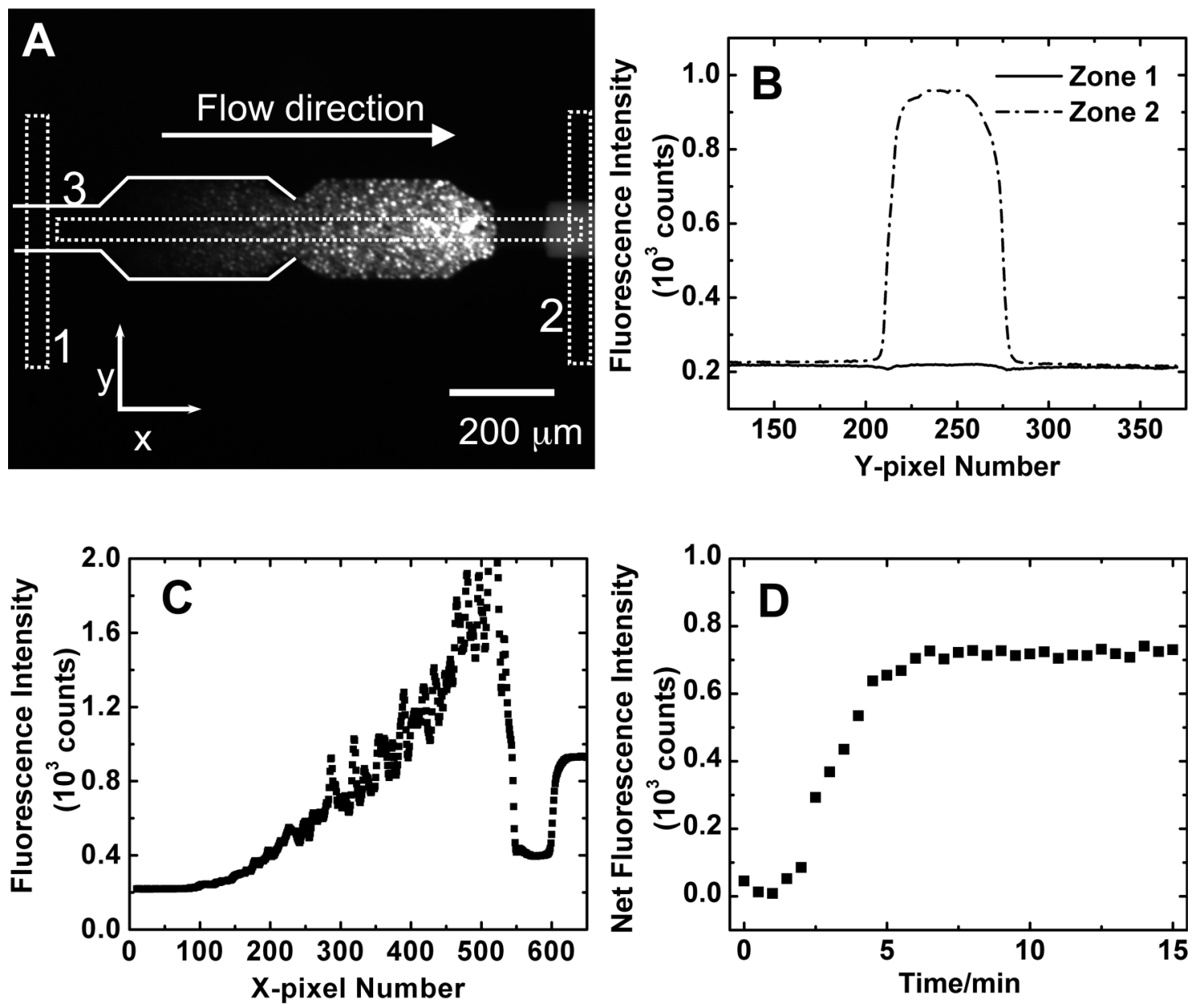

Fig. 2 (A) Fluorescence micrograph of reactor $\mathbf{I}$ obtained $15 \mathrm{~min}$ after a reactant solution consisting of $1.00 \mathrm{mM}$ glucose and $0.80 \mathrm{mM}$ Amplex Red was introduced into the reactor at a flow rate of $0.20 \mu \mathrm{L} / \mathrm{min}$. The microbeads were co-immobilized with GOx and HRP enzymes. (B) Fluorescence intensity profiles obtained from zones 1 and 2 indicated by the white dotted boxes shown in Fig. 2(A). (C) Fluorescence intensity profile obtained from zone 3 indicated by the white dotted box shown in Fig. 2(A). (D) Plot of time-dependent net fluorescence intensity. The net fluorescence intensity was obtained by subtracting the average intensity of zone 1 from that of zone 2 in the channel shown in Fig. 2(A). The $\mathrm{x}$ and y pixel dimensions are $2 \mu \mathrm{m}$, respectively.

reactor having a low aspect ratio ranging from 5-9. Accordingly, flow rates in the range of 0.05 to $0.20 \mu \mathrm{L} / \mathrm{min}$ were used for the experiments reported here.

\section{Test of the microreactors}

Products of coupled reactions shown in Scheme 2 were examined as a function of the location of the two enzymes within the microreactors. This amounts to introducing glucose (1) and Amplex Red (4) into the microreactor, and then measuring the fluorescence arising from the product (resorufin, 5) of the coupled enzyme-catalyzed reactions. The experiment was set up by introducing a $50 \mathrm{mM}$ Tris buffer solution ( $\mathrm{pH} 7.4)$ containing $1.00 \mathrm{mM}$ glucose and $0.80 \mathrm{mM}$ Amplex Red into the microreactor at a flow rate of $0.20 \mu \mathrm{L} / \mathrm{min}$. Fluorescence micrographs were collected every $30 \mathrm{~s}$ for $20 \mathrm{~min}$. Figure $2 \mathrm{~A}$ shows a typical fluorescence micrograph obtained at $15 \mathrm{~min}$ after the reactant solution was continuously pumped into the channel of reactor $\mathbf{I}$. The fluorescence-intensity profiles shown in Fig. 2B were obtained from the two zones of the microreactor enclosed within the white dotted boxes 1 and 2 in Fig. 2A. The stronger signal at zone 2 compared with zone 1 indicates the generation of a fluorescence product after the two-step sequential reactions. Figure $2 \mathrm{C}$ shows the fluorescence intensity profile obtained from the region of the microreactor enclosed within the white dotted box 3 in Fig. 2A. This intensity profile clearly shows an increase of fluorescence intensity along the flow direction, suggesting the accumulation of product from the bead bed along the flow direction in the microfluidic reactor. The fluorescence intensity measured from region 2 (x-pixel number: $>600$ ) was 4-times that measured from the region of the weir structure (x-pixel number: $550-600$ ). This fluorescenceintensity ratio reflects the height difference between the detection region $(22 \mu \mathrm{m})$ and the weir region $(6 \mu \mathrm{m})$. Figure 2D shows a graph of the time-dependent net fluorescence intensity extracted from fluorescence micrographs similar to Fig. 2A. The net fluorescence intensity was obtained by subtracting the average fluorescence intensity of zone 1 from that of zone 2 . Because the net fluorescence intensity reached a steady state within 10 min after the introduction of reactants, the data points between 10 and $15 \mathrm{~min}$ were averaged to obtain the average value of the net fluorescence intensity. A steady state fluorescence intensity can be achieved when the mass transport 

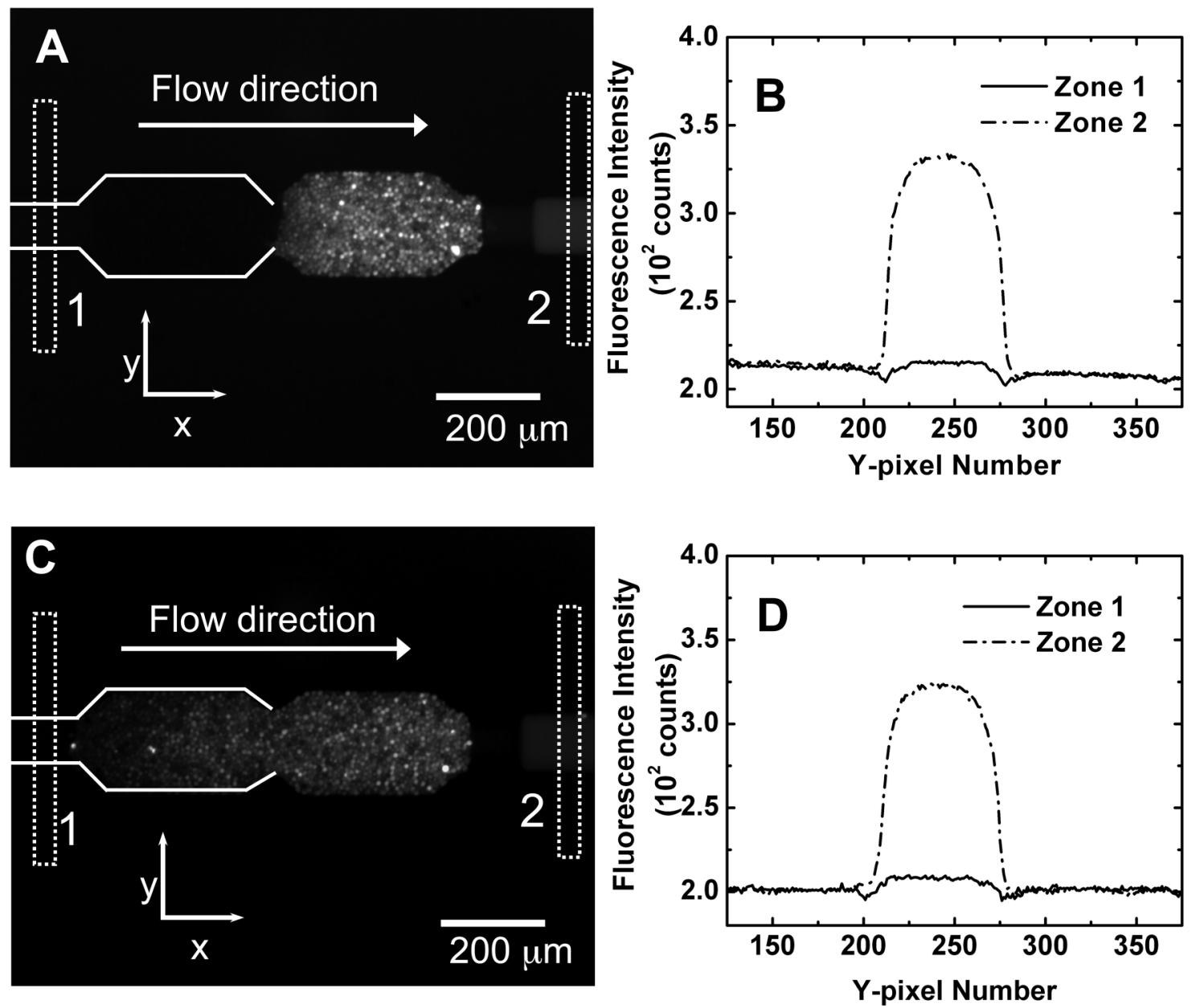

Fig. 3 (A) Fluorescence micrograph of reactor II obtained $15 \mathrm{~min}$ after a reactant solution consisting of $1.00 \mathrm{mM}$ glucose and $0.80 \mathrm{mM}$ Amplex Red was introduced into the reactor at a flow rate of $0.20 \mu \mathrm{L} / \mathrm{min}$. The left and right microchambers were packed with GOx-bearing and HRP-bearing microbeads, respectively. (B) Fluorescence intensity profiles obtained from the zones 1 and 2 indicated by the white dotted box shown in Fig. 3(A). (C) Fluorescence micrograph of reactor III obtained with the same condition as that of reactor II. GOx-bearing beads and HRP-bearing beads were mixed in a one-to-one volume ratio off-chip and packed into the two chambers. (D) Fluorescence intensity profiles obtained from zones 1 and 2 indicated by the white dotted box shown in Fig. 3(C). The $x$ and y pixel dimensions are $2 \mu \mathrm{m}$, respectively.

of reactants and products and the kinetics of immobilized enzymes reach a steady state. The time necessary to reach the steady state is a complicated function of the flow rate, mass-transfer effects of the reactant, intermediate product, and final product, and the kinetics of immobilized enzymes.

The concentrations of the fluorescent product generated from each of the microreactors were quantified using the calibration curve constructed from resorufin standard solutions (Fig. S4, Supporting Information). The average and the standard deviation of the product concentrations obtained from three-independently prepared devices were $115 \pm 4 \mu \mathrm{M}$.

In a similar way as above, reactors II and III (Scheme 1) were examined. Figures $3 \mathrm{~A}$ and $3 \mathrm{C}$ are fluorescence micrographs of reactors II and III obtained under the same experimental conditions as that of reactor I. Figures $3 \mathrm{~B}$ and $3 \mathrm{D}$ show the fluorescence intensity profiles obtained from zones 1 and 2 in Figs. 3A and 3C, respectively. In the case of reactor II fluorescence emission was not observed in the first chamber of the sequential reactor, because the beads bearing enzymes for the second step of the sequential enzyme reactions were present only in the second chamber. On the contrary, reactor III emitted fluorescence in both of the chambers, as shown in Fig. 3C, because the two different sets of beads bearing each of the GOx and HRP enzymes were mixed and packed into all chambers. The fluorescence intensities observed from region 2 of reactors II and III were seven-times weaker than that from region 2 of reactor I. The steady-state product concentrations produced from reactors II and III were $4.5 \pm 0.2 \mu \mathrm{M}(n=3)$ and $4.8 \pm$ $0.2 \mu \mathrm{M}(n=3)$, respectively. The overall reaction efficiency of these two reactors were $\sim 25$-times lower than that of reactor $\mathbf{I}$. Note that the fluorescence intensity of resorufin shows a non-linear relationship with the resorufin concentration at a certain concentration range (Fig. S4(B), Supporting Information). There was no significant difference in the reaction efficiency between reactors II and III.

The better overall reaction efficiency of reactor I compared with reactors II and III can be explained as follows. The first point is the importance of a mass-transport effect of the intermediate during the sequential, two-step reaction. In reactor I, the GOx and HRP enzymes were co-immobilized in close 

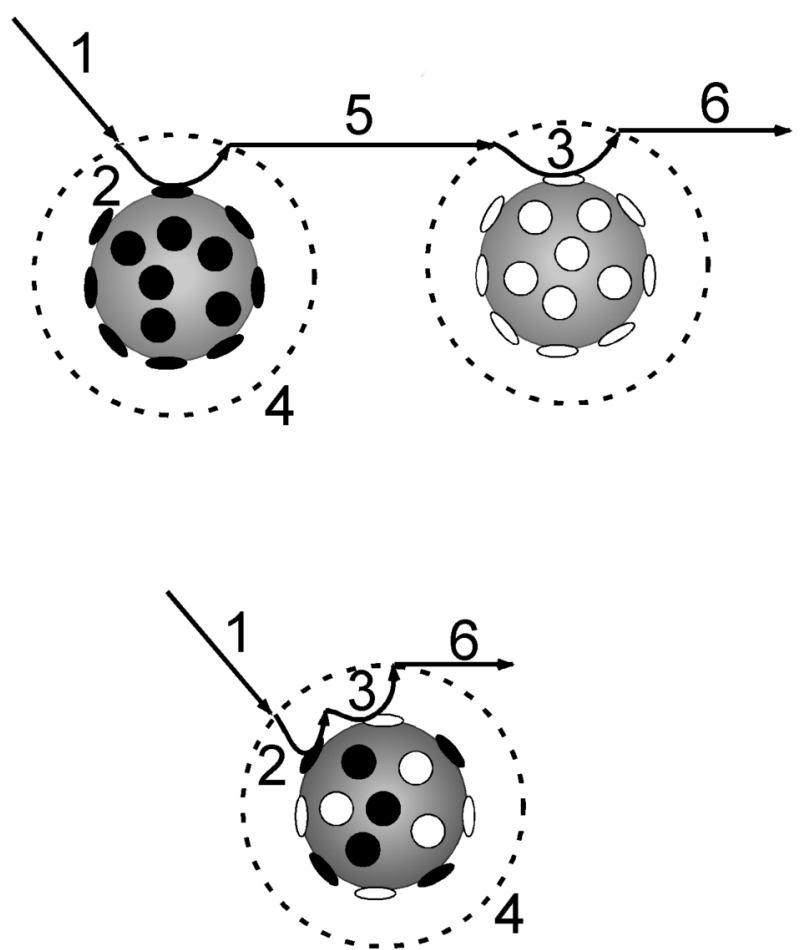

Scheme 3 Schematic diagrams showing the effect of mass-transport of an intermediate product on the sequential enzyme reactions. The black and white circles indicate GOx and HRP enzymes, respectively. 1. mass-transport of a reactant, 2. GOx-catalyzed reaction, 3. HRPcatalyzed reaction, 4. diffusion layer, 5. mass-transport of an intermediate product, 6 . mass-transport of a final product.

proximity to each other on the bead surfaces, and therefore the intermediate product, which was used as a substrate for the second enzyme reaction, could be efficiently converted to a final product without any significant diffusional loss of the intermediate product, as illustrated in Scheme 3. Digital simulation results confirmed the experimental results. ${ }^{29}$ While an intermediate product was almost non-existent in reactor $\mathbf{I}$ with the introduction of $1 \mathrm{mM}$ reactant at a flow rate of $0.2 \mu \mathrm{L} / \mathrm{min}, \sim 160 \mu \mathrm{M}$ of product was estimated to be produced from reactor I. This indicates a fast conversion of the intermediate product to the final product in reactor $\mathbf{I}$ without significant diffusional loss of the intermediate product. On the other hand, the simulation results of both reactors II and III showed that $\sim 150 \mu \mathrm{M}$ of the intermediate product along with $\sim 10 \mu \mathrm{M}$ of the product was generated from both reactors under the same simulation condition as reactor $\mathbf{I}$, suggesting a notable diffusional depletion of the intermediate product in both reactors II and III. A similar phenomenon is observed in nature. ${ }^{23,24}$ Cells employ intracellular metabolic pathways based on multi-step enzyme reactions. To increase the overall reaction efficiency, a spatiotemporal control of the intermediate product is essential..$^{30}$ A cellular system optimizes the compartmentalization of metabolic pathways to reduce any significant loss of an intermediate product.

Secondly, reactor I was less likely to undergo inhibition of GOx enzyme sites by $\mathrm{H}_{2} \mathrm{O}_{2}$, an intermediate product generated from the GOx-catalyzed reaction of glucose. ${ }^{31}$ It was reported that the stability of the GOx enzyme could be greatly improved by adding catalase enzymes to remove $\mathrm{H}_{2} \mathrm{O}_{2}$ quickly. ${ }^{32}$ Thus,

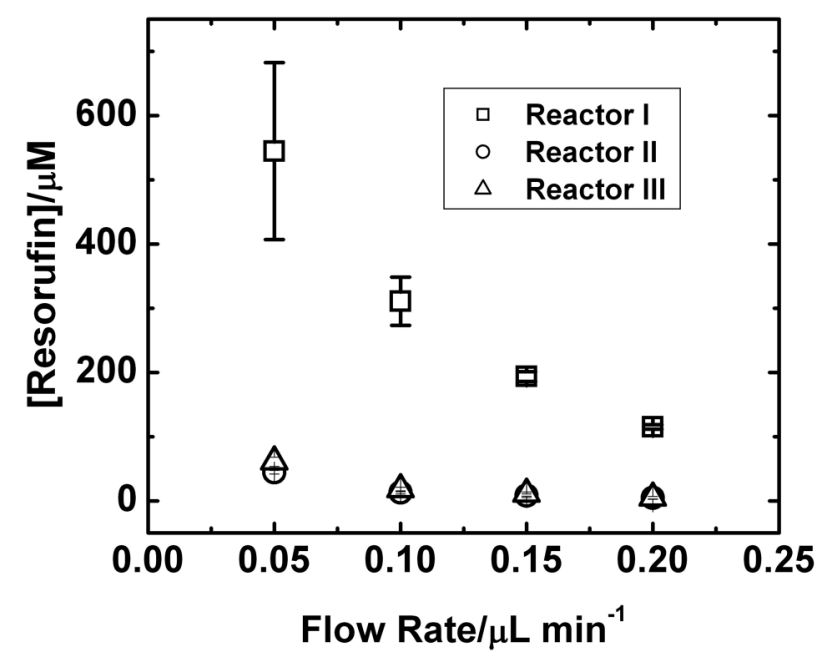

Fig. 4 Plot of the flow rate-dependent concentrations of the resorufin product determined from reactors I, II, and III. The error bar represents $1 \sigma$ of three measurements obtained from three independently prepared microfluidic devices.

there is less chance of inhibiting GOx enzymes by the intermediate product in reactor $\mathbf{I}$ than in the other two types of reactors, because the intermediate product can be quickly converted to the final product by having HRP enzymes nearby GOx in reactor $\mathbf{I}$. After rinsing the three microreactors with a buffer solution extensively, we examined whether they can be reused. The products were produced only $10-50 \%$ as much as those generated in the first-time use of the reactors, which suggests that they cannot be reused.

Next, we examined the flow-rate effect on the reactor efficiency. Here, the reactor efficiency was defined as the ratio of the product concentration to the reactant concentration at a given flow rate, and expressed as $\%$. The final product concentrations were plotted as a function of the flow rate, as shown in Fig. 4. In general, the product concentration was inversely proportional to the flow rate regardless of the reactor configurations. When the flow rate decreases, the residence time of substrates in a beads-packed reactor can be prolonged, thus making more contact time between the substrate molecules and the enzymes. The data also indicates that the two-step enzyme reactions occurring on the beads are not mass-transfer limited. The overall reaction efficiency of reactor $\mathbf{I}$ could reach $-50 \%$ when the flow rate was reduced to $0.05 \mu \mathrm{L} / \mathrm{min}$. On the contrary, the reaction efficiencies of reactors II and III were $<10 \%$ at a flow rate of $0.05 \mu \mathrm{L} / \mathrm{min}$. This indicates that reactor I shows much better efficiency than the other two reactors irrespective of the flow rate.

\section{Conclusions}

In summary, we showed that the microfluidic reactor could be an essential platform for examining the overall reaction efficiency of multi-step, sequential enzyme reactions. The configuration of a microfluidic reactor could be easily modified using simple microfabrication technology and microbeads as a supporting matrix for immobilizing enzymes. Furthermore, a microfluidic reactor based on immobilized enzymes can be used to mimic the cellular metabolic pathways that employ sequential reactions catalyzed by multiple enzymes, because different 
enzymes required for the sequential reactions can be easily localized in designated places in a microfluidic reactor. This platform will be useful to devise new synthetic pathways in vivo and screen engineered enzyme pairs for better conversion efficiency. Finally, it is also necessary to develop a theoretical model for quantitatively describing the kinetics of two-step enzyme reactions in a microfluidic reactor. This theoretical work of ours will be reported soon. ${ }^{29}$

\section{Acknowledgements}

Support from the Research Foundation at SUNY College at Buffalo is greatly appreciated.

\section{Supporting Information}

Procedures for microfabrication, immobilizing enzymes on microbeads, and determining the binding capacity of avidincoated beads for biotin-GOx and biotin-HRP along with Figs. S1-S4 were included in the Supporting Information. This material is available free of charge on the Web at http:// www.jsac.or.jp/analsci/.

\section{References}

1. R. DiCosimo, J. McAuliffe, A. J. Poulose, and G. Bohlmann, Chem. Soc. Rev., 2013, 42, 6437.

2. Y. Asanomi, H. Yamaguchi, M. Miyazaki, and H. Maeda, Molecules, 2011, 16, 6041.

3. J. Krenkova and F. Foret, Electrophoresis, 2004, 25, 3550.

4. H. Zhou, Z. Ning, F. Wang, D. Seebun, and D. Figeys, FEBS J., 2011, 278, 3796.

5. Q. Zhang, X. Tang, F. Hou, J. Yang, Z. Xie, and Z. Cheng, Anal. Biochem., 2013, 441, 51.

6. C. Berne, L. Betancor, H. R. Luckarift, and J. C. Spain, Biomacromolecules, 2006, 7, 2631.

7. P. Lin, S. Zhao, X. Lu, F. Ye, and H. Wang, J. Sep. Sci., 2013, 36, 2538.

8. Y. Gao, R. Zhong, J. Qin, and B. Lin, Chem. Lett., 2009, $38,262$.

9. T. Honda, M. Miyazaki, Y. Yamaguchi, H. Nakamura, and H. Maeda, Lab Chip, 2007, 7, 366.
10. S. Kundu, A. S. Bhangale, W. E. Wallace, K. M. Flynn, C. M. Guttman, R. A. Gross, and K. L. Beers, J. Am. Chem. Soc., 2011, 133, 6006.

11. S. Matosevic, G. J. Lye, and F. Baganz, J. Biotechnol., 2011, 155, 320.

12. M. S. Thomsen, P. Poelt, and B. Nidetzky, Chem. Commun (Cambridge, U. K.), 2007, 2527.

13. P. He, G. Greenway, and S. J. Haswell, Microfluid. Nanofluid., 2010, 8, 565.

14. M. B. Kerby, R. S. Legge, and A. Tripathi, Anal. Chem., 2006, 78,8273 .

15. G. H. Seong, J. Heo, and R. M. Crooks, Anal. Chem., 2003, 75,3161 .

16. A. Guiseppi-Elie, N. F. Sheppard, S. Brahim, and D. Narinesingh, Biotechnol. Bioeng., 2001, 75, 475.

17. C. N. Monty, I. Oh, and R. I. Masel, IEEE Sens. J., 2008, 8 , 580.

18. J. Heo and R. M. Crooks, Anal. Chem., 2005, 77, 6843.

19. M. A. Holden, S. Y. Jung, and P. S. Cremer, Anal. Chem., 2004, 76, 1838.

20. M.-Y. Lee, A. Srinivasan, B. Ku, and J. S. Dordick, Biotechnol. Bioeng., 2003, 83, 20.

21. H. Mao, T. Yang, and P. S. Cremer, Anal. Chem., 2002, 74 , 379.

22. G. H. Seong and R. M. Crooks, J. Am. Chem. Soc., 2002, 124,13360

23. K. Mosbach and B. Mattiasson, Acta Chem. Scand., 1970, 24, 2093.

24. K. Mosbach, Sci. Am., 1971, 224, 26.

25. T. Buranda, J. Huang, V. H. Perez-Luna, B. Schreyer, L. A. Sklar, and G. P. Lopez, Anal. Chem., 2002, 74, 1149.

26. D. Losic, J. G. Shapter, and J. J. Gooding, Langmuir, 2002 , 18,5422 .

27. C. Leung, C. Xirouchaki, N. Berovic, and R. E. Palmer, Adv. Mater., 2004, 16, 223.

28. M. A. Holden, S. Kumar, A. Beskok, and P. S. Cremer, $J$. Micromech. Microeng., 2003, 13, 412.

29. J. Heo, submitted, 2014.

30. C. M. Agapakis, P. M. Boyle, and P. A. Silver, Nat. Chem. Biol., 2012, 8, 527.

31. J. Bao, K. Furumoto, M. Yoshimoto, K. Fukunaga, and K. Nakao, Biochem. Eng. J., 2003, 13, 69.

32. M. Yoshimoto, Y. Miyazaki, M. Sato, K. Fukunaga, R. Kuboi, and K. Nakao, Bioconjugate Chem., 2004, 15, 1055. 